\title{
Culture of adult mouse neurons
}

\author{
Lars Eide and Cynthia T. McMurray
}

BioTechniques 38:99-104 (January 2005)

\begin{abstract}
Primary neuronal cells used to model physiology are generally limited to embryonic tissue. However, embryonic tissue is not optimal as a model for age-related changes in physiology or late-onset disease. Successful culturing of neurons from adult animals, however, has been historically difficult, if not impossible. Here, we report methodology for routine and reliable cultivation of healthy striatal neurons from adult mice. The new methodology is cost-effective and improves the speed and simplicity of neuronal isolation.
\end{abstract}

\section{INTRODUCTION}

Tissues from both embryonic and early postnatal pups have been used to successfully cultivate primary neurons from different regions of the brain (1). Few glia are present at these early developmental stages, and at plating, almost pure neuronal cultures can be obtained. Although embryonic neurons represent an easy and effective source of primary neurons, their developmental stage is not always appropriate for the topic of interest. For example, neurodegeneration often occurs later in life and can arise from age-dependent decreases of mitochondrial function, accumulation of DNA damage, or increases in oxidative stress $(2,3)$. The apparent inability to culture healthy neurons from adult animals has precluded these types of analyses.

In order to overcome this barrier, we have identified isolation, growth, and attachment parameters that might, in part, explain the poor success rate of culturing adult neurons. By optimizing conditions, we report here a reliable method to obtain healthy neuronal cultures from adult animals. The new methodology is based on simple culture conditions using standard dispersal methods without special isolation procedures, preparation of a glial feeding layer, the use of special growth media, or the addition of growth factors. This procedure has advantages over a previously described protocol (4), in that it improves ease, speed, and simplicity of the preparation while reducing cost.

\section{MATERIALS AND METHODS}

\section{Buffers and Materials}

Twenty-five milligrams papain (Worthington Biochemical, Lakewood, NJ, USA) was dissolved in HEPESbuffered saline (HBS) to $2 \mathrm{mg} / \mathrm{mL}$ and kept at $4^{\circ} \mathrm{C}$ for up to 2 months. HBS, containing $10 \mathrm{mM}$ HEPES [N-(2-hydroxyethyl)-piperazine- $\mathrm{N}^{\prime}$ (2-ethanesulfonic acid)], $145 \mathrm{mM}$ $\mathrm{NaCl}, 22 \mathrm{mM} \mathrm{KCl}, 5 \mathrm{mM}$ glucose, $\mathrm{pH}$ 7.3, was always filter-sterilized before use. Plating media comprised of Dulbecco's modified Eagle medium (DMEM) supplemented with high glucose (Invitrogen, Carlsbad, CA, USA), $10 \%$ fetal calf serum (FBS), 10\% F-12 (Invitrogen), penicillin (100 $\mathrm{U} / \mathrm{mL})$, and streptomycin $(100 \mu \mathrm{g} / \mathrm{mL})$. Feeding media comprised B27 neurobasal media (Invitrogen) supplemented with penicillin $(100 \mathrm{U} / \mathrm{mL})$ and streptomycin $(100 \mu \mathrm{g} / \mathrm{mL})$. Glutamine was added to the media on the day of use by diluting frozen stocks to a final concentration of $2 \mathrm{mM}$.

\section{Polyornithine Coating of Culture Dishes}

Plastic culture dishes were coated with L-polyornithine prior to use. Each 35-mm plate was incubated overnight in $2.0 \mathrm{~mL}$ (or $0.5 \mathrm{~mL}$ for 24-dish plate) of $0.5 \mathrm{mg} / \mathrm{mL}$ borate buffer $(10 \mathrm{mM}$ $\mathrm{Na}_{2} \mathrm{~B}_{4} \mathrm{O}_{7}, \mathrm{pH}$ 8.4). After incubation, plates were washes twice with deionized water prior to use.

\section{Isolation of Neurons}

The experimental protocol was approved by the Mayo Clinic Institutional Animal Care and Use Committee. C57BL/6 mice of 1.0-1.5 years were anesthetized by ether prior to cervical dislocation. Neurons from two regions of the brain were tested, and the method was successfully used for either. Striatum or cortical tissue was isolated under sterile conditions, rinsed in HBS, and minced into small pieces. The minced tissue (approximately 0.5 $\mathrm{mL}$ ) was then treated with $1 \mathrm{~mL}$ papain for $15 \mathrm{~min}$ at $37^{\circ} \mathrm{C}$. The papain/HBS supernatant was removed and replaced with $4-6 \mathrm{~mL}$ of prewarmed plating medium $(1.5 \mathrm{~mL} / 35-\mathrm{mm}$ dishes and 0.5 $\mathrm{mL} / 16-\mathrm{mm}$ dish), after which the tissue was immediately triturated $(20-30$ times with a $1-\mathrm{mL}$ pipet). The average yield during isolation was $7.4 \pm 1 \times 10^{5}$ (SD) cells per animal (quantified from five different isolations) and is comparable to that reported previously for hippocampal neurons (4).

The dispersed cells were counted and adjusted to maximum of $3.5 \times 10^{5}$ cells/ $\mathrm{mL}$. Typically, $2 \mathrm{~mL}$ of this cell solution was added to each $35-\mathrm{mm} \mathrm{L-polyor-}$ nithine-coated dish, or alternatively, $0.5 \mathrm{~mL}$ for a $16-\mathrm{mm}$ dish. Cells were 
allowed to settle for $15 \mathrm{~min}$, washed once, and replaced with prewarmed plating medium (1.5 $\mathrm{mL} / 35-\mathrm{mm}$ dish and $0.5 \mathrm{~mL} / 16-\mathrm{mm}$ dish). After plating, cells were incubated at $37^{\circ} \mathrm{C}$ in $5 \% \mathrm{CO}_{2}$ and incubated for 11 days without media replacement. On day 11, the plating media was replaced with the same volume of feeding medium. The average number of attached cells was $2.3 \pm 0.8 \times 10^{5}(\mathrm{SD})$ cells $/ \mathrm{mL}$ or about $60 \%$ of the plated cells. Survival was optimal in 1- to 6-mm dishes and was typically observed to the middle of each dish. The middle area constitutes about $8 \mathrm{~mm}$ in a $16-\mathrm{mm}$ dish or $25 \%$ of the dish area. Cell images were taken by a digital camera mounted on a Nikon TMS-12807 microscope (Nikon, Melville, NY, USA) with a $20 \times$ lens.

\section{Assessment of Mitochondrial Membrane Potential}

Mitochondrial potential in isolated brain cells was evaluated using 5,5, 6', $6^{\prime}$-tetrachloro-1, $1^{\prime}, 3,3^{\prime}$-tetraethylbenzimidazolcarbo-cyanine iodide (JC-1; $1 \mu \mathrm{g} / \mathrm{mL}$ ). JC-1 distribution depends on mitochondrial potential. JC-1 can be free or in complexes in the mitochondria. The distribution of free and complexed JC-1 was determined by flow cytometry analysis using a FACScan ${ }^{\mathrm{TM}}$ (BD Biosciences Immunocytometry Systems, San Jose, CA, USA) with 488-nm excitation laser. When complexed with polarized mitochondria, the emission maximum of the dye is different than in its free form (580 versus $510 \mathrm{~nm}$ ). Average emitted light intensity values of the complexed (em 585/42 nm) and free (em 530/30 $\mathrm{nm}$ ) form of JC-1 (FL2 ave and FL1 ${ }_{\text {ave }}$, respectively) were identified using the WinMDI 2.8 software (Scripps Research Institute, La Jolla, CA, USA). FL1 and FL2 signals were gated above 10 to minimize noise. The fraction of complexed JC-1 was quantified as $\mathrm{FL} 2_{\mathrm{ave}} /\left(\mathrm{FL} 2_{\mathrm{ave}}+\mathrm{FL} 1_{\mathrm{ave}}\right)$ and gave the best reproducible estimate of mitochondrial membrane potential (MMP). This ratio was found to be insensitive to 10fold variation in JC-1 concentration. Cells were depolarized with $1 \mu \mathrm{M}$ valinomycin after each measurement to verify that the "depolarization ratio" of JC-1 (= 0.2) was similar in each experiment. Three independent replicates of the MMP measurements and autofluorescence were combined to generate the detailed kinetic plot with different time points measured each time. The time points in separate experiments reproduces a high quality curve.

Isolated mitochondria were prepared as previously described (5). Mitochondria respiration buffer comprised $210 \mathrm{mM}$ sucrose, $20 \mathrm{mM}$ $\mathrm{KCl}, 3 \mathrm{mM}$ glycyl-glycine, $1 \mathrm{mM}$ $\mathrm{KH}_{2} \mathrm{PO}_{4}, 1$ mM EGTA, pH 7.2, and contained $0.3 \mu \mathrm{g} / \mathrm{mL} \mathrm{JC}-1$. MMP in these organelles was analyzed by flow cytometry using the same method described for cells. Reactions with the indicated respiratory substrates were measured at room temperature. Mitochondria were charged with substrates for complex I (malate and pyruvate; $1.5 / 3 \mathrm{mM}$ ) and for complex II (succinate; $1 \mathrm{mM}$ ). Reversibility of the MMP was demonstrated by inhibiting complex I activity (with rotenone; $0.5 \mu \mathrm{M}$ ) and complex III activity (with antimycinA; $10 \mu \mathrm{g} / \mathrm{mL}$ ).

\section{Reactive Oxygen Species Production and Redox Balance}

Reactive oxygen species (ROS) production was assayed by staining isolated cells with $10 \mu \mathrm{M}$ dihydrochlorofluorescein (DHCF) diacetate prior to flow cytometry analysis. Oxidized DHCF was recorded in FL1 (ex/em: 488/530-580). Autofluorescence (FL1 detection) in the isolated brain cell samples is an estimate of the redox balance (6) and recorded at 530-580 $\mathrm{nm}$ with the excitation at $488 \mathrm{~nm}$.

\section{RESULTS}

We evaluated three parameters that might influence viability and distinguish differences between adult and embryonic neuronal cultures. Cultures were tested for differences in their bioenergetic properties, for effects induced by the physical isolation procedure, and for alterations in their growth conditions.

\section{Bioenergetic Parameters in Isolated Brain Cells}

Cells require functional mitochondria for energy production and growth. Mitochondrial integrity is evident by the degree of MMP, which correlates with production of ROS (7). Higher than normal ROS can be harmful to cells. Therefore, we tested if MMP in adult neurons was compromised relative to embryonic cells and affected their viability in culture. To measure MMP, we isolated striatal and cortical brain cells from 17-day old embryos (E17) or from adult animals of $1.0-1.5$ years. If reduced viability of adult cells was due to compromised mitochondria, we expected that adult cells would have an abnormally high polarization state, and MMP would be greater relative to embryonic brain cells in culture.

Figure 1. Cellular parameters in isolated brain cells. (A) Mitochondrial membrane potential (MMP) as a function of time. MMP was measured as ratio of complexed 5,5,6', $6^{\prime}$-tetrachloro-1,1',3,3'-tetraethylbenzimidazolcarbocyanine iodide (JC-1) in brain cell suspensions. Mitochondrial depolarization with valinomycin results in JC-1 ratio of 0.2. (B) Time-dependent reactive oxygen species (ROS) production was detected as fluorescence of cells stained with dihydrochlorofluorescein (DHCF)-diacetate. (C) Increase in autofluorescence of cells with time. Circles, adult brain cells; triangles, embryonic brain cells. 
The degree of MMP was measured using a fluorescent dye, JC-1. JC-1 can be used to detect MMP, since it will form a complex with mitochondria only if they are polarized and healthy. JC-1 can detect MMP because it emits light at different wavelengths depending on its polarization state; $580 \mathrm{~nm}$ when complexed with mitochondria, and 510 $\mathrm{nm}$ when it is free. Thus, the energetic properties were assessed by measuring the intracellular ratio of complexed to free forms of JC-1 in solution. We found that both adult and embryonic neurons acquired the same degree of MMP with time in solution. However, adult cells were capable of a more efficient MMP buildup and reached a steady-state level after approximately $50 \mathrm{~min}$ in medium (Figure 1A). These data suggested the possibility that adult cells might generate more ROS. To directly test this possibility, we independently measured

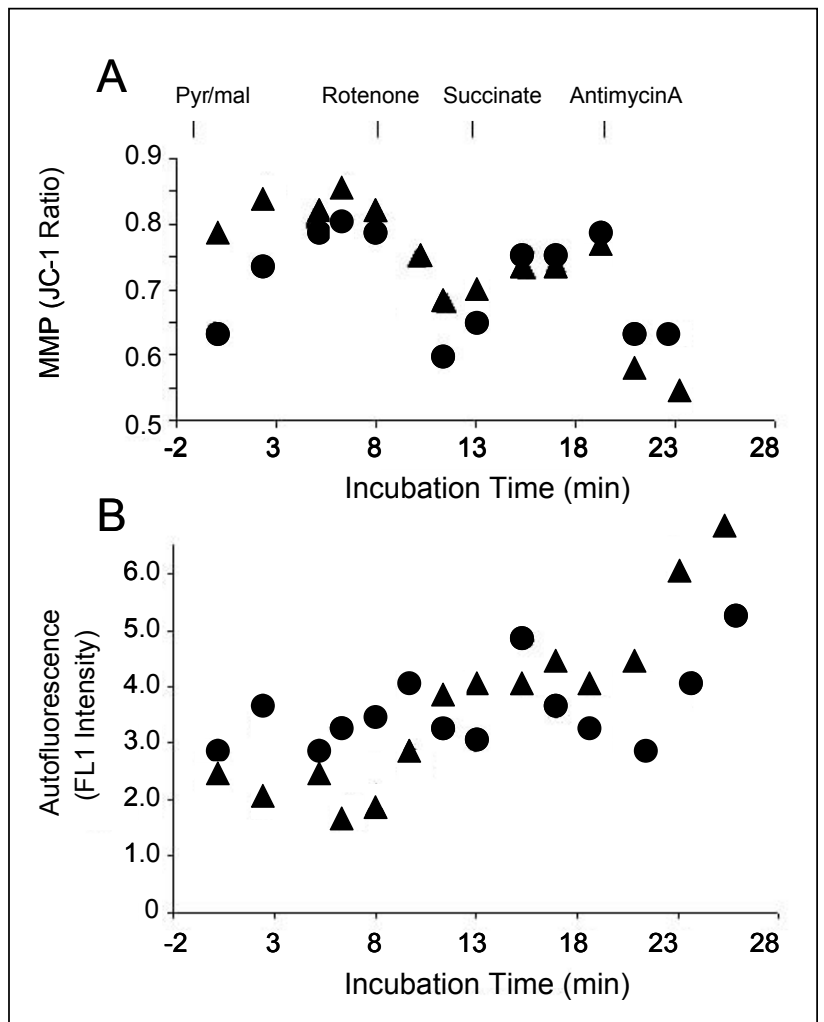

Figure 2. Mitochondrial membrane potential (MMP) and autofluorescence in isolated mitochondria. (A) MMP of isolated mitochondria incubated in respiration buffer prior to addition of $\mathrm{Pyr} / \mathrm{mal}(1.5 / 3 \mathrm{mM})$, rotenone $(0.5 \mu \mathrm{M})$, succinate $(1 \mathrm{mM})$, and antimycinA $(10 \mu \mathrm{g} / \mathrm{mL})$ as indicated. (B) Parallel autofluorescence measurement (measured by flow cytometry in FL1 channel) of respiring mitochondria treated as in panel A, but without 5,5, $6^{\prime}, 6^{\prime}$-tetrachloro-1,1',3,3'-tetraethylbenzimidazolcarbocyanine iodide (JC-1). Mitochondria were isolated from adult brains (circles) and embryonic brains (triangles).
ROS using a ROS-sensitive fluorescent dye DHCF. However, we found that the ROS level in adult brain cells was significantly less than in embryonic brain cells (Figure 1B). Therefore, the differences in viability of adult and embryonic neuronal cultures could not be ascribed to ROS production.

The absence of a correlation between ROS correlation and survival did not clude other processes associated with Intracellular redox potential is determined by ratio of reducing compounds (NADH and $\mathrm{FADH}_{2}$ ) to oxidized compounds (NAD ${ }^{+}$, FAD). Since reducing compounds are necessary (hablish MM, the redox ratio directly correlates with MMP. The level of FAD provides a good estimate of the redox ratio in a cell and can be readily detected measuring the cellular autofluorescence recorded at 500-530 nm. Although other groups in the cell (including protein residues) are autofluorescent, mitochondrial FAD has been reported to be the major contributor (8). Indeed, autofluorescence of dispersed adult cells was remarkably weaker than dispersed embryonic cells (Figure 1C). These data raised the possibility that the decreased FAD content in adult neurons might arise from an inability of mitochondria to properly respire and oxidize normal substrates.

To test this hy $\mathrm{p}$ ot he s i s, we isolated mitochondria from adult and embryonic brain tissue and measured MMP during respiration (Figure 2A). MMP is reversible; it increases using the appropriate substrates, and it decreases by treatment with mitochondrial complex inhibitors. When mitochondria were manipulated to reversibly polarize and depolarize (Figure 2A), no differences between the two culture types were detected. Consistent with these results, the redox ratios in the respiring mitochondria were indistinguishable (Figure 2B). Based on these data, it was unlikely that the observed metabolic difference in embryonic and adult brain originated from changes in mitochondrial function or was a critical factor in cell survival. Therefore, we focused attention on the physical condition of the isolation procedure.

\section{Attachment of Isolated Brain Cells}

During isolation of brain cells, some cell lysis occurs and inevitably produces debris. Cell lysis can change the growth medium by lowering the $\mathrm{pH}$ or by releasing cytotoxic agents (glutamate), and both can have deleterious influence on survival of the remaining cells (4). Further debris can interfere with cell attachment. Both of these problems are easily overcome by washing. However, neurons develop processes relatively slowly, and 
washing can remove neurons if they are not attached. Therefore, we measured the time required for attachment for adult neuronal cultures to optimize cell survival and to improve yield.

We measured optimum attachment time by assaying the cell density the in supernatant above the poly-L-ornithinecoated surface in an incubation dish. We found that the number of unattached cells in the media dropped initially to a level $(40 \%)$ and did not change significantly within the next 4-5 h (Figure 3A). It was possible that the drop in the number of unattached cells was due to cell death. To control for this, we maintained the cells in medium in the absence of a poly-ornithinylated surface. After $5 \mathrm{~h}, 70 \%$ of these unattached cells excluded trypan blue, indicating that they were living (grey circle in Figure 3A). Thus, many adult brain cells were not dying after dispersal, but they did not always attach efficiently.

In additional experiments, we confirmed that cells able to attach do so within the first $10 \mathrm{~min}$. Therefore, in the culturing procedure (Figure 3B), media was withdrawn from cells by aspiration 15 min after plating, and the cells washed once before further incubation in plating medium. Using this procedure, growth of adult neurons was detected after approximately 1 week in culture. For adult cells, no neurons were detected in dishes that were either left unwashed or washed $2 \mathrm{~h}$ after plating, demonstrating the importance of removing debris.

We also tested whether cell survival and attachment of unwashed cells could be improved by incubating them before dispersal in the presence of agents that suppress cell death pathways. However, incubation with calcium chelators such as EGTA (1 mM), ifenprodil ( $5 \mu \mathrm{M}$; antagonist of NMDA receptor subunit), z-VAD (50 $\mu \mathrm{M}$; suppresses caspase activation), 3-aminobenzamide $[1 \mathrm{mM}$; inhibitor of poly(ADP) polymerase] to suppress oxidative stress-induced cell death, and DNase $(1 \mathrm{U} / \mathrm{mL}$; to inhibit DNAmediated sticking of cell to debris) had no effect on cell survival. No neurons were detected in unwashed cultures after any of these manipulations. Cholesterol has been demonstrated to have a beneficial effect on neurons in the absence of glia (9). However, we also found that cholesterol $(5 \mu \mathrm{g} / \mathrm{mL})$ had no significant effect on survival (data not shown).

Since the dispersal procedure required trituration and papain treatment, it was possible that the isolation procedure itself might be differentially harmful to the adult cells. However, neither of these parameters had any visible effects on cell survival. The absence of protease treatment resulted in dirty cultures containing glial cells, but no neurons survived after plating (Figure 4A). Thus, the papain treatment was essential. In fact, prolonged treatment with proteases resulted in cleaner culture, likely due to degradation of cellular debris (Figure 4, B and C). The yield of living cells in Figure 4, B and C, was similar and within normal variation of compa-

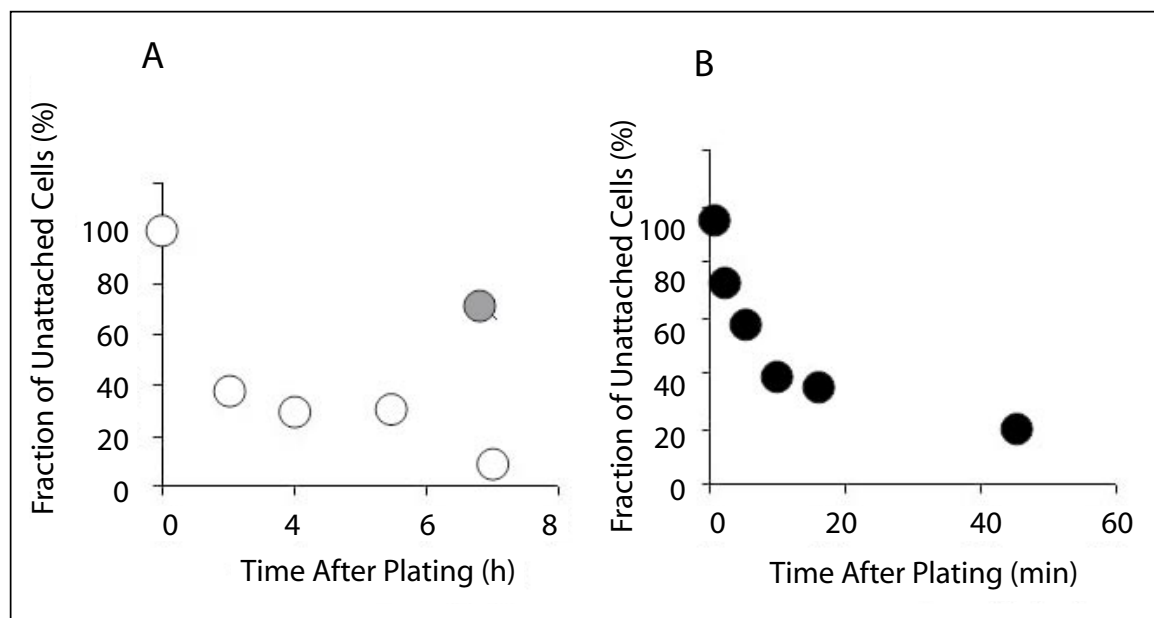

Figure 3. Kinetics of cell attachment. Fraction of unattached cells as a function of plating time. (A) Longer periods after plating, between $0-8 \mathrm{~h}$. (B) Shorter periods up to $1 \mathrm{~h}$ after plating. Grey circle indicates the fraction of cells that survived a prolonged time in isolation mixture without being plated onto poly-L-ornithine-coated dishes. Samples were taken during the incubation, and cells were counted on a Hy-Lite Hemacytometer (0.1 mm deep; Hausser Scientific, Horsham, PA, USA).

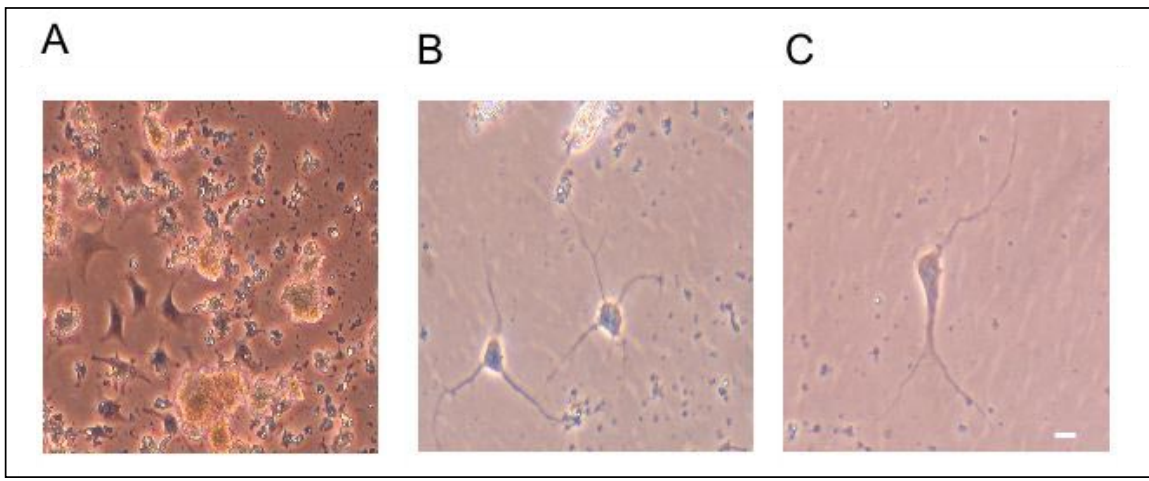

Figure 4. Purity of neuronal cell culture depends on effective protease treatment. Images are dispersed striatal cells after 5 days in culture when cells were isolated using the described methodology: (A) without the use of papain before plating; (B) with 5 min of papain treatment before plating; or (C) after two 10-min papain treatments before plating. Scale bar indicates $20 \mu \mathrm{m}$. Cell images were taken by a digital camera mounted on a Nikon TMS-12807 microscope with a $20 \times$ lens. 
either parameter significantly enhanced survival. Neurons were cultured in a 24-dish plate with $0.5 \mathrm{~mL} / \mathrm{dish}$. After 1 week, "colonies" of adult neurons appeared. Neuronal growth did not require the preparation of a glial feeding layer (1). Rather, neurons clustered together and grew on top of a glial layer, which formed naturally from cells present in the dispersed solution before plating (Figure 5A). Notably, neurons developed primarily in the middle of the dish, where the medium level was lowest and the local concentration of excreted factors would be highest. In fact, we found that the concentration of secreted factors was a critical parameter. While growth media for embryonic neuronal cultures typically replaces plating media after 3-4 days in culture (when neuronal processes have begun to develop), the same replacement induced cell death in adult cultures (Figure 5B). Adult neurons survived best when they were maintained for longer periods without media replacement. Survival was also improved when a higher density of cells was cultured. Using these procedures, we were able to successfully culture, for 4 weeks, adult neurons from 1 - to 1.5-year-old animals. A representative culture derived from a 1-year-old $\mathrm{C} 57 \mathrm{bl} / 6$ mouse is shown (Figure 5A).

\section{DISCUSSION}

The apparent inability of adult neurons to be cultivated compared to embryonic neurons prompted an investigation of important parameters that might influence cell viability. Here, we report that healthy cultures of adult neurons can be successfully isolated and maintained when attention is paid to the isolation conditions. The most critical parameters that improved cell survival were those relating to the physical isolation and the growth conditions. We found that nicely developing neurons from adult mice can be obtained by effective protease treatment prior to plating, followed by careful washing of the attached cells. Finally, it is essential to maintain a low medium level above the cells and/or plate at high density. Brewer and colleagues have reported that addition of trophic factors such as fibroblast growth factor-
2 (FGF2) can be beneficial for survival (4). Furthermore, specialized media with altered osmolarity may limit neuronal death of adult neurons, especially during medium replacement (4). While survival might be improved by these modifications, the simple culturing conditions described in this report allow good yields of healthy adult neurons with fewer steps, less labor, and at lower costs.
Mitochondria are involved in many different cellular processes related to cell homeostasis, and we observed that the kinetics of MMP build-up were distinctly different in adult versus embryonic cultures. Based on FAD measurements measured in isolated mitochondria, the elevated MMP in adult cells coincides with a lower intracellular redox ratio in mitochondria. 


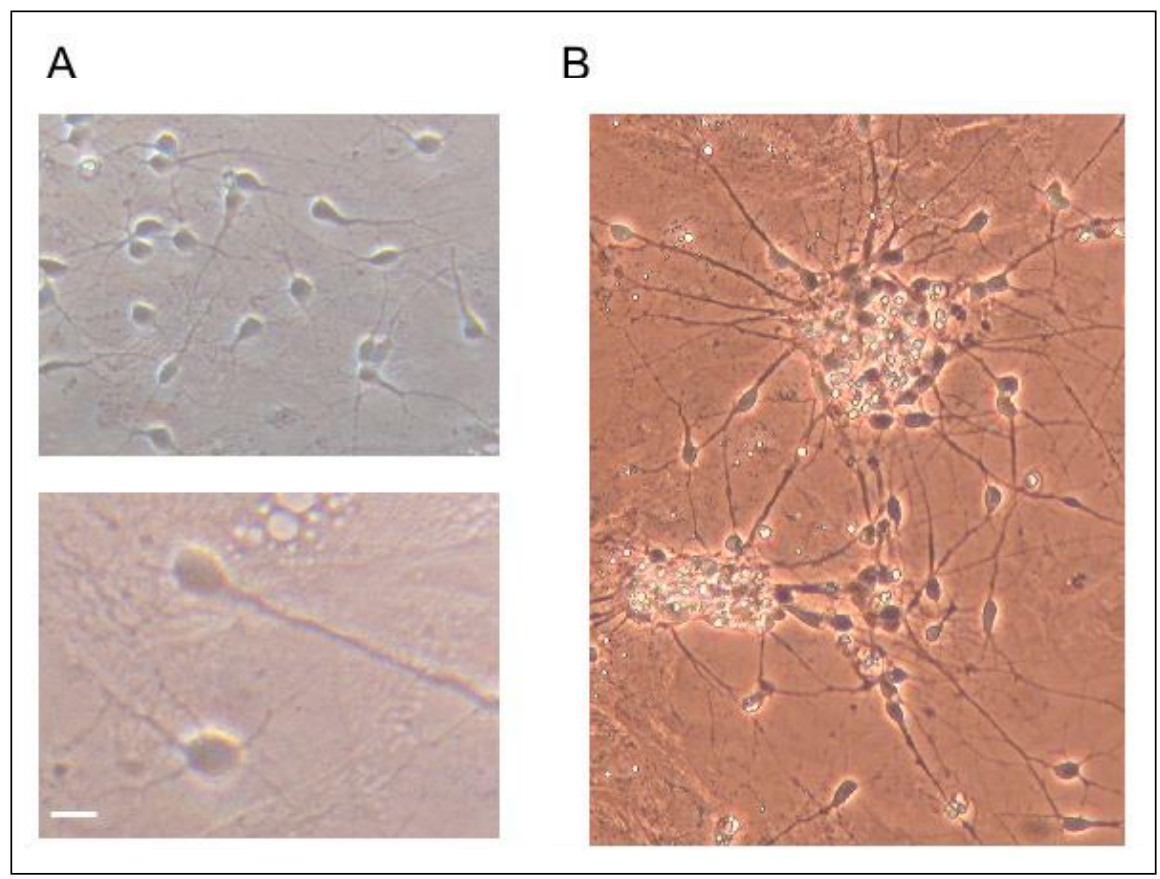

Figure 5. Adult neurons in culture. Brain cells from the striatum of 1-year-old bl6 mice were cultured as described. (A, top) Neuronal growth of dispersed striatal cultures containing both neurons and glia after 20 days in culture. (A, bottom) Magnification ( $2 \times$ ) of the same cultures. (B) Some neuronal death can occur after medium replacement and is observed as bright bodies. Scale bar indicates $20 \mu \mathrm{m}$. Cell images were taken by a digital camera mounted on a Nikon TMS- 12807 microscope with a $20 \times$ lens.
Survival of axotomized neonatal retinal ganglion cells is optimal in a mildly reducing environment (10). More severe reducing conditions can also impair survival (10). Therefore, it is possible that adult brain cells isolated in a sufficiently low redox state limits attachment and growth, and low MMP contributes either directly or indirectly to improve survival and attachment of adult neuronal cultures.

\section{ACKNOWLEDGMENTS}

We are grateful to Drs. Echson Holmuhamedov and Eugenia Trushina for excellent advice and suggestions during different aspects of this work. L.E. receives financial support from the Norwegian Research Council (FUGE). This work was supported by the Mayo Clinic Rochester, National Institutes of Health grants NS40738 (C.T.M.), and the Hereditary Disease Foundation (C.T.M.).

\section{COMPETING INTERESTS STATEMENT}

The authors declare no competing interests.

\section{REFERENCES}

1.Banker, G. 1998. Culturing Nerve Cells, 2nd ed. Cellular and Molecular Neuroscience. MIT Press, Cambridge.

2.Beal, M.F. 1995. Aging, energy, and oxidative stress in neurodegenerative diseases. Ann. Neurol. 38:357-366.

3.Schapira, A.H. 1996. Oxidative stress and mitochondrial dysfunction in neurodegeneration. Curr. Opin. Neurol. 9:260-264.

4.Brewer, G.J. 1997. Isolation and culture of adult rat hippocampal neurons. J. Neurosci. Methods 71:143-155.

5.Anderson, M.F. and N.R. Sims. 2000. Improved recovery of highly enriched mitochondrial fractions from small brain tissue samples. Brain Res. Brain Res. Protoc. 5:95-101.

6.Van de, W.M. and D. Pipeleers. 1983. Autofluorescence-activated cell sorting of pancreatic islet cells: purification of insulin-containing B-cells according to glucose-induced changes in cellular redox state. Biochem. Biophys. Res. Commun. 114:835-842.

7.Starkov, A.A. and G. Fiskum. 2003. Regulation of brain mitochondrial $\mathrm{H} 2 \mathrm{O} 2$ production by membrane potential and $\mathrm{NAD}(\mathrm{P}) \mathrm{H}$ redox state. J. Neurochem. 86:1101-1107.

8.Pavlova, I., K. Sokolov, R. Drezek, A. Malpica, M. Follen, and R. Richards-Kortum. 2003. Microanatomical and biochemical origins of normal and precancerous cervical autofluorescence using laser-scanning fluorescence confocal microscopy. Photochem. Photobiol. 77:550-555.

9.Mauch, D.H., K. Nagler, S. Schumacher, C. Goritz, E.C. Muller, A. Otto, and F.W. Pfrieger. 2001. CNS synaptogenesis promoted by glia-derived cholesterol. Science 294:1354-1357.

10.Geiger, L.K., K.R. Kortuem, C. Alexejun, and L.A. Levin. 2002. Reduced redox state allows prolonged survival of axotomized neonatal retinal ganglion cells. Neuroscience 109:635-642.

Received 2 June 2004; accepted 14 September 2004.

Address correspondence to:

Cynthia T. McMurray

Dept. Molecular Pharmacology and

Experimental Therapeutics

Mayo Clinic Rochester

Rochester, MN 55905, USA

e-mail: mcmurray.cynthia@mayo.edu 